\title{
Efficacy of a Physical Training Program on Pregnancy Related Lumbopelvic Pain
}

\author{
Seyedeh Tahereh Mirmolaei ${ }^{1}$, Noureddin Nakhostin Ansari ${ }^{2,3,4}$, Mahmood Mahmoudi $^{5}$, Fahimeh Ranjbar ${ }^{6,1^{*}}$
}

\begin{abstract}
Objectives: A large number of women suffer from lumbopelvic pain during pregnancy. This pain continues in considerable percentage of women after pregnancy and causes considerable distress and disability. The purpose of this study was to investigate the efficacy of a physical training program on lumbopelvic pain and its physical disability during pregnancy.

Materials and Methods: A quasi-experimental study was performed to investigate the effects of a physical training program in women with lumbopelvic pain in 2010-2011. A total of 180 pregnant women were randomly assigned to the intervention $(\mathrm{n}=90)$ and control groups $(\mathrm{n}=90)$. The physical training program consisted of stretching and strengthening exercises and body posture modifications. This 12-week program was administered to the intervention group only and the control group received the routine prenatal care. The main outcome for measurement were pain and physical disability that was assessed at baseline and 12 weeks post treatment by visual analog scale (VAS) and Oswestry Disability Index, respectively.

Results: There was no significant difference in pain intensity and physical disability between groups before intervention $(P>0.05)$, however pain and physical disability decreased significantly in the intervention group $(P<0.05)$.

Conclusions: It seems that a designed physical program and body postures modification may reduce the severity of lumbopelvic pain and its related disability in pregnant women. It is recommended that midwives emphasize the importance of back pain and pelvic pain in prenatal counseling sessions and provide solutions for pain relief, including physical exercise and posture modification. Keywords: Low back pain, Pelvic pain, Pregnancy, Physical training, Exercise, Disability
\end{abstract}

\section{Introduction}

Back pain is the most common musculoskeletal problem in pregnant women $(1,2)$. It is estimated that $50 \%$ to $70 \%$ of the pregnant women suffer from this problem (3-5). Pregnancy-related lumbopelvic pain is a progressive pain that affects different aspects of women's life including work, housekeeping, free time activities and even sleeping(1). This pain can trigger chronic back pain and in varying periods after pregnancy it can cause considerable distress and disability (6). Reasons for the higher prevalence of back pain in pregnant women are fatigue, increased mobility of joints due to the hormonal changes imposed to collagen, pressure from the growing fetus, weight gain, and increased load on the spine that changes body posture (7). Despite measures taken to treat pregnancyrelated lumbopelvic pain, the pain intensity is increased by pregnancy progressing. Although it is highly unlikely to prevent the pregnancy low back pain, research suggests that reducing the pain and improving its complications is possible (8). Using the ultrasound as well as superficial heating is contraindicated during pregnancy. Electrical stimulation should not be used in lower back, abdomen, hips, and pelvis to avoid the possibility of potential contact with the fetus. The effect of electrical current on the fetus is controversial. In general, there is also limited evidence about the protective efficacy of lumbosacral binders (9). It seems that the exercise programs, regardless of their severity or combination can be helpful in reducing the pain (10). However there is no strong evidence on the effect of exercise therapy on prevention and treatment of pregnancy-related lumbopelvic pain, and therefore more studies are required $(11,12)$. In addition to training the pregnant woman in prenatal classes, the midwives are also responsible for their physical preparation (13). Encouraging pregnant women in healthy behaviors such as physical activities and exercises is difficult in pregnancy due to physiological barriers such as fatigue, nausea and weight gain. Regular appointment with health care provider throughout the pregnancy is a unique opportunity for women to benefit from healthy behaviors for themselves and their fetuses (14). Health care providers should be aware of interventions that are safe and effective to minimize the pain during pregnancy (15). Most of the women report their concern such as low back pain to healthcare providers but unfortunately their pain has not been managed well (16). Pregnancy related low

Received 10 December 2016, Accepted 9 July 2017, Available online 5 August 2017

${ }^{1}$ Department of Midwifery and Reproductive Health, Tehran University of Medical Sciences, Tehran, Iran. ${ }^{2}$ Department of Physiotherapy, School of Rehabilitation, Tehran University of Medical Sciences, Tehran, Iran. ${ }^{3}$ Sports Medicine Research Center, Neuroscience Institute, Tehran University of Medical Sciences, Tehran, Iran. ${ }^{4}$ Neuromusculoskeletal Research Center, Iran University of Medical Sciences, Tehran, Iran. ${ }^{5}$ Department of Epidemiology and Biostatistics, School of Public Health, Tehran University of Medical Sciences, Tehran, Iran. ${ }^{6}$ Nursing Care Research Center, Iran University of Medical Sciences, Tehran, Iran.

*Corresponding Author: Fahimeh Ranjbar, Nursing Care Research Center, Iran University of Medical Sciences, Rashid Yasemi St., Valiasr Ave., P.O. Box 19395-4798, Tehran, Iran. Tel/Fax+98 21 88671613, Email: ranjbar.f@iums.ac.ir 
back pain is common in Iranian women $(3,17,18)$. The high prevalence of lumbo- pelvic pain during pregnancy in Iranian women requires more attention by researchers and health care providers (3). Therefore, this study was accomplished to determine the effect of a physical training program on pregnancy-related lumbopelvic pain.

\section{Material and Methods \\ Study Design}

A quasi-experimental study was performed to investigate the effect of a physical training program on the women with lumbopelvic pain during 2010-2011.

\section{Participants}

Women were included in the study if they were between 18 to 35 years old, were in the gestational week between 17 and 22, and had singleton pregnancy.

Exclusion criteria included the absolute or relative contraindications for exercise in pregnancy according to guidelines of American College of Gynecologist and Obstetrics (19), history of surgery, fracture or disease of spinal column and pelvis, inflammatory disease or rheumatoid arthritis, history of recent abdominal surgery, threatened abortion, absence of patients in training classes, and censoring performing physical training exercises less than 3 times a week.

\section{Outcome Measurement}

The main outcome to be measured was pain and physical disability that was assessed by visual analog scale (VAS) and Oswestry Disability Index, respectively. The VAS is a quick and quite easy-to-use user-friendly instrument for rating pain. The validity and reliability of VAS have been established before (20). The VAS is a $10 \mathrm{~cm}$ line with descriptors at each end (from none to severe), along which respondents place a mark indicating their subjective pain. The score is measured as the distance of the mark from zero end of the line.

There is not a gold standard for evaluating disability in low back pain, however Oswestry Disability Questionnaire is a reliable scale to detect improvement or worsening in most subjects (21). The questionnaire was developed by Fairbank et al and consists of 10 items of pain, lifting, self-care ability, the ability to walk, ability to sit, sexual function, ability to stand, social life, sleep quality, and ability to travel (22). Each item is scored from $0-5$, with higher values representing greater disability. The total score would be multiplied by 2 and expressed as a percentage. Zero means no physical disability and 100 means the maximum disability. Persian version of Oswestry questionnaire was found to be a reliable and valid measure of physical disability (3).

\section{Intervention}

The 12-week exercise program was administered to the intervention group only, and the control group received the routine prenatal care. The training program which was developed by an expert physiotherapist included a prenatal education class about simple anatomy, physiological changes in pregnancy, factors causing low back pain, proper posture in lying, sitting and standing, proper lifting techniques, and specific exercises. The exercises consisted of stretching and strengthening such as pelvic tilting, knee pull, Kegel exercise, wall squats, adductor stretch, pelvic elevation, pelvic rotation, arm and leg raise. Women were encouraged to perform each exercise 10 times a day for 12 weeks. The hold times for strengthening and stretching exercises were 3 and 20 seconds, respectively. The rest time between the exercises was 6 seconds. The participants were asked to perform the exercises daily according to the manual. It took 20 minutes for women to perform all the exercises. The women were requested to record their daily exercise on the exercise sheet. Women in the intervention group were encouraged to continue the exercise program at home by weekly phone call from researcher.

\section{Procedure}

The participants were recruited from two prenatal clinics of Lolagar and Najmieh hospitals in Tehran, Iran. The study subjects were randomly assigned into 2 groups of intervention and control. At the beginning, demographic data including the age, height and weight were recorded.

Women were asked to determine the intensity of their pain using VAS and complete the Oswestry disability Questionnaire. Measurements were taken at baseline and after treatment.

\section{Statistical Analysis}

The statistical analysis of the data using mean, range, frequency and distribution, chi square test, independent and paired $t$ test were performed. The data were analyzed using the SPSS software version 16.0 and $P$ values $<0.05$ were considered significant. The demographic and clinical data were assessed by independent $t$ tests or chi square as appropriate. The paired $t$ test was used to analyze withingroup changes.

\section{Results}

Of 190 women eligible for the study, 171 women were selected for the study. Figure 1 shows the subjects flow and reasons for loss to follow-up. The data from 171 women were used for analysis. The baseline data (Table $1)$ indicates that the women in both groups did not differ $(P<0.05)$.

The pain intensity did not differ statistically between groups at baseline. However, a statistically significant difference in favor of the intervention group was observed in the pain intensity. The pain increased in the control group and decreased in the intervention group. The difference between the mean of pain intensity score before and after intervention was significant (Table 2).

Physical disability did not differ statistically between 


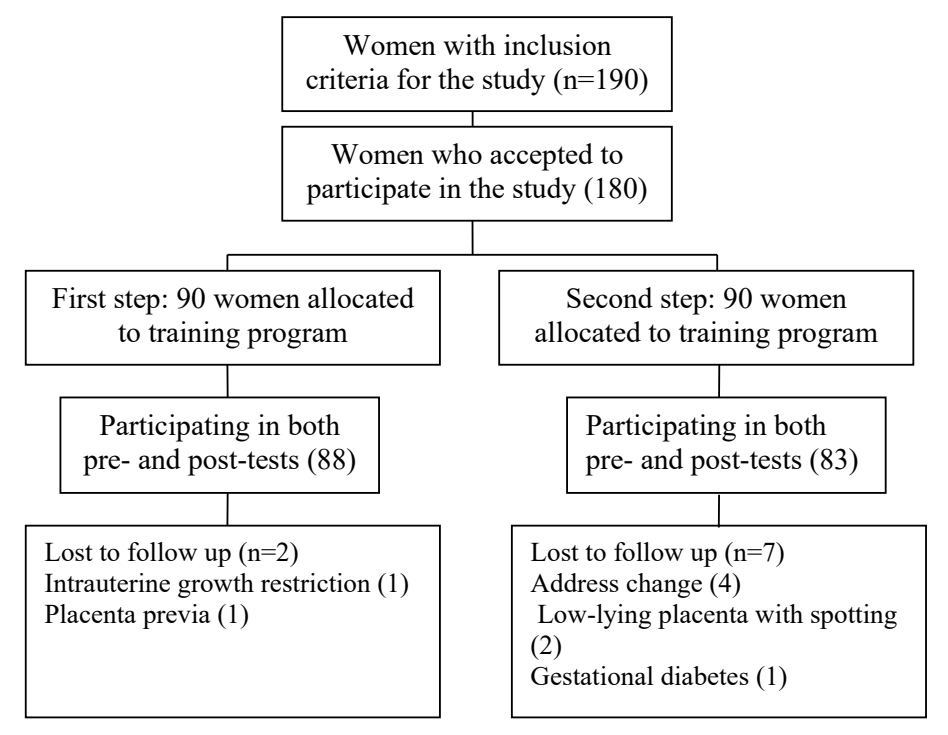

Figure 1. Flowchart of the Study

groups at baseline, but Independent $t$ tests showed significant difference between the physical disability scores of the intervention and control groups after intervention $(P<0.05)$.

The results of paired sample $t$ test showed a significant difference in the mean of the physical disability in the intervention and control groups before and after the intervention $(P<0.018, P<0.05)$. Independent $t$ tests showed that differences between the pre-test and posttest physical disability scores of experimental and control groups were significant $(P<0.05)$ (Table 3$)$.

\section{Discussion}

Our findings showed that there was a significant difference between the intervention and control groups in terms of pain intensity which is due to mean pain intensity increase in the control group. The results also showed that physical disability due to lumbopelvic pain decreased in the intervention group and increased in the control group significantly. Therefore, it can be concluded that a designed physical program may reduce the severity of lumbopelvic pain and its related disability in pregnant women. Likewise, several studies have confirmed the positive effects of exercises on the pain intensity in low back and pelvic region. Shim et al designed a program including 6 physical exercises for 32 pregnant women who complained of the back pain (23). Their exercise program included pelvic tilting, knee pull, straight leg raising, curl up, lateral straight leg raising, and the Kegel exercise which was very similar to the program in the present study. Similar to findings of the present study, implementing the program significantly reduced the lumbar and pelvic pain intensity and physical limitation compared to the control group. In South Africa, Kluge et al investigated the effect of strengthening and stretching exercises on the pain intensity of women with pregnancy-related low back pain (24). Their particular exercise program reduced the pain intensity of women with low back pain and pelvic girdle

Table 1. Characteristics of Participants in the Beginning of the Study

\begin{tabular}{|c|c|c|c|}
\hline Variable & Intervention Group & Control Group & $P$ Value \\
\hline Age, mean $( \pm S D)$ & $26.46(3.93)$ & $25.56(3.54)$ & $0.954^{a}$ \\
\hline BMI, mean ( $\pm S D)$ & $3.93(23.97)$ & $23.63(3.89)$ & $0.489^{a}$ \\
\hline Gestational age, mean ( \pm SD) & $19.04(2.07)$ & $19.03(2.10)$ & $0.820^{\mathrm{a}}$ \\
\hline Low back pain (\%) & 44.6 & 34.1 & $0.160^{\mathrm{b}}$ \\
\hline Pelvic girdle pain (\%) & 61.4 & 75 & $0.057^{\mathrm{b}}$ \\
\hline Low back and pelvic pain (\%) & 26.5 & 25 & $0.822^{\mathrm{b}}$ \\
\hline Employment (\%) & 12 & 12.5 & $0.928^{\mathrm{b}}$ \\
\hline History of back pain before pregnancy (\%) & 28.9 & 38 & $0.199^{b}$ \\
\hline History of back pain during menstruation (\%) & 52 & 58 & $0.657^{\mathrm{b}}$ \\
\hline History of taking OCP (\%) & 15.7 & 21.6 & $0.321^{\mathrm{b}}$ \\
\hline Reporting pain to prenatal care provider (\%) & 59.7 & 46.7 & $0.113^{b}$ \\
\hline
\end{tabular}

Abbreviation: OCP, oral contraceptive pills.

${ }^{a} t$ test; ${ }^{b}$ Chi-square test. 
Table 2. Comparing Mean Pain Intensity Changes Between and Within Groups

\begin{tabular}{lccl}
\hline Pain Intensity & $\begin{array}{c}\text { Control Group } \\
\text { Mean( } \pm \text { SD) }\end{array}$ & $\begin{array}{c}\text { Intervention Group } \\
\text { Mean( } \pm \text { SD) }\end{array}$ & $\begin{array}{l}\text { Independent } \\
\text { t-test Result }\end{array}$ \\
\hline $\begin{array}{l}\text { Before } \\
\text { intervention }\end{array}$ & $3.56(2.71)$ & $3.87(2.76)$ & 0.604 \\
$\begin{array}{l}\text { After } \\
\text { intervention }\end{array}$ & $5.01(3.08)$ & $2.94(2.39)$ & $<0.001$ \\
$\begin{array}{l}\text { Change rate } \\
\begin{array}{l}\text { Paired } t \text { test } \\
\text { result }\end{array}\end{array}$ & $1.44(2.45)$ & $-0.84(3.15)$ & $<0.001$ \\
\hline
\end{tabular}

Table 3. Comparing Mean Physical Disability Changes Between and Within Groups

\begin{tabular}{lccc}
\hline Disability & $\begin{array}{c}\text { Control Group } \\
\text { Mean (士SD) }\end{array}$ & $\begin{array}{c}\text { Intervention Group } \\
\text { Mean (士SD) }\end{array}$ & $\begin{array}{c}\text { Independent } \\
\text { t-test Result }\end{array}$ \\
\hline $\begin{array}{l}\text { Before } \\
\text { intervention }\end{array}$ & $20.95(14.23)$ & $18.83(14.64)$ & 0.338 \\
$\begin{array}{l}\text { After } \\
\text { intervention }\end{array}$ & $26.14(18.53)$ & $16.2(12.55)$ & $<0.001$ \\
$\begin{array}{l}\text { Change rate } \\
\begin{array}{l}\text { Paired } t \text { test } \\
\text { result }\end{array}\end{array}$ & $5.18(12.03)$ & $-2.80(10.87)$ & $<0.001$ \\
\hline
\end{tabular}

pain. Nevertheless, in this study only woman with lumbar or pelvic pain were recruited. The training program in the present study was implemented with a preventive approach for all the pregnant women. Therefore, the present study focused on the effect of an exercise program on the pain prevention.

In accordance with the results of the present study, Garshasbi and Faghih Zadeh showed reduction in low back pain intensity of pregnant women with exercise during the second trimester of the pregnancy (25). They investigated the effect of an aerobic exercise program including one-hour aerobic exercise, 3 times per week for 12 weeks during pregnancy on the intensity of low back pain in Tehran, Iran. The only tool to evaluate the effect of exercise program on pain intensity was the Quebec functional index (KEBEK) in this study. Indeed the pain intensity was measured indirectly through physical disability. The KEBEK was significantly decreased in the exercise group after intervention, but in the control group, it was increased.

In relation to the prevention of lumbopelvic pain during pregnancy, these findings were in agreement with a study conducted by Morkved et al (26) who reported that lumbopelvic pain in the training group was significantly less than that in the control group. The scores of functional status were also significantly higher in the training group. This may be due the pain prevention in the training group.

Contrary to findings of the present study, Stafne et al concluded that regular exercise during pregnancy had no effect on the prevalence of lumbopelvic pain (27). They conducted a randomized control trial to study the effect of aerobic and strengthening exercises on lumbopelvic pain.
Self-reports of lumbopelvic pain was not significantly different at 36 weeks between the groups of exercise and standard prenatal care. Their data were analyzed according to the "intention-to-treat" principle and the study population in this study consisted of pregnant women with lumbopelvic pain, who contributed to higher report of lumbopelvic pain. Furthermore, weekly group training was conducted by physiotherapists and women were encouraged to do the home exercises twice a week. It seems the greater number of exercises and more supervision on home-based instructions is needed for positive effect of program on pain.

Miquelutti et al designed a birth preparation program with an intervention protocol including physical exercises, educational activities and instructions on home-based exercises. (28). Inconsistently this protocol failed to control the lumbopelvic pain and the authors concluded that lumbopelvic pain during pregnancy requires greater attention in birth preparation programs. Early pregnancy prenatal classes provide an opportunity for giving advice to pregnant women on how to properly rest and perform activities, predicting physical changes during pregnancy, preventing or relieving common pregnancy problems such as back pain, improving post-delivery recovery and preventing gynecologic and orthopedic problems in the future (13). Although general physical exercises or fitness classes are beneficial for women during pregnancy (29), but it seems that regular prenatal exercise has no effect on pregnancy-related lumbopelvic pain (21). It is better that specific lumbo- pelvic exercise to be integrated in birth preparation programs.

One of the limitations of this study was home exercises without supervision. Although home-based instructions for exercise can save time and money, monitoring the proper implementation of these exercises is very important. Many pregnant women fear exercising, so encouraging them to physical activity and exercise is so difficult because of physiological changes and fear of harm to baby. In this study, the only way to make sure of doing the exercises by the women were their declaration and the data sheet they filled, so the women might not follow the exercises according to the given instructions. For this limitation, the training program was performed at least once under the supervision of the coach in educational class, meanwhile we provided the participants with the training manual to minimize the errors. Furthermore, in order to make sure of doing the exercises regularly and completely, women were followed by weekly phone calls, and asked to do exercises completely. Another limitation was related to poor contribution of hospitals to birth preparation classes in the present study. As only two hospitals contributed in the study, then it is not possible to generalize the results to all pregnant women.

\section{Conclusions}

In conclusion, a physical training program and body 
postures modification during pregnancy can decrease the lumbopelvic pain and disability. It is recommended that midwives emphasize the importance of back pain and pelvic pain in prenatal counseling and provide solutions for pain relief, including physical exercise and posture modification. It seems that specific training programs for lumbopelvic pain can be more beneficial in reducing pain and disability of pregnant women. For reducing lumbopelvic pain and disability, the specific exercise programs should be integrated with birth preparation programs, fitness classes, or general prenatal exercises.

Different programs are designed to reduce the low back pain and pelvic girdle pain in pregnancy, but there are controversies in the results of the studies. Further studies are needed to confirm the effects of the exercise programs on low back pain and pelvic pain during the pregnancy.

\section{Conflict of Interests}

The authors have no conflict of interests to disclose.

\section{Ethical Issues}

The study was approved by the Research Council of Nursing and Midwifery School, Tehran University of Medical Sciences. All the women signed the informed consent form to take part in this study.

\section{Financial Support}

This study was supported by a grant from Tehran University of Medical Sciences.

\section{Acknowledgments}

We thank our colleagues in Lolagar and Najmie hospitals for their effective cooperation in this study.

\section{References}

1. Wang SM, Dezinno P, Maranets I, Berman MR, Caldwell-Andrews AA, Kain ZN. Low back pain during pregnancy: prevalence, risk factors, and outcomes. Obstet Gynecol. 2004;104(1):65-70. doi:10.1097/01. AOG.0000129403.54061.0e

2. Wang SM. Backaches related to pregnancy: the risk factors, etiologies, treatments and controversial issues. Curr Opin Anaesthesiol. 2003;16(3):269-273.

3. Mousavi SJ, Parnianpour M, Vleeming A. Pregnancy related pelvic girdle pain and low back pain in an Iranian population. Spine (Phila Pa 1976). 2007;32(3):E100-104. doi:10.1097/01.brs.0000254123.26649.6e

4. Mogren IM, Pohjanen AI. Low back pain and pelvic pain during pregnancy: prevalence and risk factors. Spine (Phila Pa 1976). 2005;30(8):983-991. doi:10.1097/01. brs.0000158957.42198.8e

5. Olsson C, Nilsson-Wikmar L. Health-related quality of life and physical ability among pregnant women with and without back pain in late pregnancy. Acta Obstet Gynecol Scand. 2004;83(4):351-357. doi:10.1111/j.00016349.2004.00384.x

6. Perkins J, Hammer RL, Loubert PV. Identification and management of pregnancy-related low back pain. J Nurse
Midwifery. 1998;43(5):331-340. doi:10.1016/S00912182(98)00032-9

7. Mantle J, Haslam J, Barton S. Physiotherapy in Obstetrics and Gynaecology. 2nd ed. China: Butterworth Heinemann Co; 2004.

8. Perkins J, Hammer RL, Loubert PV. Identification and management of pregnancy-related low back pain. J Nurse Midwifery. 1998;43(5):331-340.

9. Borg-Stein J, Dugan SA, Gruber J. Musculoskeletal aspects of pregnancy. Am J Phys Med Rehabil. 2005;84(3):180-192.

10. Pennick V, Liddle SD. Interventions for preventing and treating pelvic and back pain in pregnancy. Cochrane Database Syst Rev. 2013(8):Cd001139. doi:10.1002/14651858.CD001139.pub3

11. Stuge B, Hilde G, Vollestad N. Physical therapy for pregnancy-related low back and pelvic pain: a systematic review. Acta Obstet Gynecol Scand. 2003;82(11):983-990. doi:10.1034/j.1600-0412.2003.00125.x

12. Gutke A, Betten C, Degerskar K, Pousette S, Olsen MF. Treatments for pregnancy-related lumbopelvic pain: a systematic review of physiotherapy modalities. Acta Obstet Gynecol Scand. 2015;94(11):1156-1167. doi:10.1111/ aogs. 12681

13. Fraser DM, Cooper MA. Myles Textbook for Midwives. Edinburg: Churchil Livingstone; 2003.

14. Wadsworth P. The Benefits of Exercise in Pregnancy. J Nurse Pract. 2007;3(5):333-339. doi:10.1016/j.nurpra.2007.03.002

15. Chang HY, Jensen MP, Lai YH. How do pregnant women manage lumbopelvic pain? Pain management and their perceived effectiveness. J Clin Nurs. 2015;24(9-10):13381346. doi:10.1111/jocn. 12742

16. Pierce H, Homer CS, Dahlen HG, King J. Pregnancy-related lumbopelvic pain: listening to Australian women. Nurs Res Pract. 2012;2012:387428. doi:10.1155/2012/387428

17. Mohseni-Bandpei MA, Fakhri M, Ahmad-Shirvani M, et al. Low back pain in 1,100 Iranian pregnant women: prevalence and risk factors. Spine J. 2009;9(10):795-801. doi:10.1016/j.spinee.2009.05.012

18. Ansari NN, Hasson S, Naghdi S, Keyhani S, Jalaie S. Low back pain during pregnancy in Iranian women: Prevalence and risk factors. Physiother Theory Pract. 2010;26(1):4048. doi:10.3109/09593980802664968

19. Artal R, O’Toole M. Guidelines of the American College of Obstetricians and Gynecologists for exercise during pregnancy and the postpartum period. Br J Sports Med. 2003;37(1):6-12; discussion 12.

20. Wewers ME, Lowe NK. A critical review of visual analogue scales in the measurement of clinical phenomena. Res Nurs Health. 1990;13(4):227-236. doi:10.1002/nur.4770130405

21. Fritz JM, Irrgang JJ. A comparison of a modified Oswestry Low Back Pain Disability Questionnaire and the Quebec Back Pain Disability Scale. Phys Ther. 2001;81(2):776-788. doi:10.1093/ptj/81.2.776

22. Fairbank JC, Couper J, Davies JB, O’Brien JP. The Oswestry low back pain disability questionnaire. Physiotherapy. 1980;66(8):271-273.

23. Shim MJ, Lee YS, Oh HE, Kim JS. Effects of a backpain-reducing program during pregnancy for Korean women: a non-equivalent control-group pretest-posttest study. Int J Nurs Stud. 2007;44(1):19-28. doi:10.1016/j. ijnurstu.2005.11.016 
24. Kluge J, Hall D, Louw Q, Theron G, Grove D. Specific exercises to treat pregnancy-related low back pain in a South African population. Int J Gynaecol Obstet. 2011;113(3):187-191. doi:10.1016/j.ijgo.2010.10.030

25. Garshasbi A, Faghih Zadeh S. The effect of exercise on the intensity of low back pain in pregnant women. Int J Gynaecol Obstet. 2005;88(3):271-275. doi:10.1016/j. ijgo.2004.12.001

26. Morkved S, Salvesen KA, Schei B, Lydersen S, Bo K. Does group training during pregnancy prevent lumbopelvic pain? A randomized clinical trial. Acta Obstet Gynecol Scand. 2007;86(3):276-282. doi:10.1080/00016340601089651

27. Stafne SN, Salvesen KA, Romundstad PR, Stuge B, Morkved
S. Does regular exercise during pregnancy influence lumbopelvic pain? A randomized controlled trial. Acta Obstet Gynecol Scand. 2012;91(5):552-559. doi:10.1111/ j.1600-0412.2012.01382.x

28. Miquelutti MA, Cecatti JG, Makuch MY. Evaluation of a birth preparation program on lumbopelvic pain, urinary incontinence, anxiety and exercise: a randomized controlled trial. BMC Pregnancy Childbirth. 2013;13:154. doi:10.1186/1471-2393-13-154

29. Nascimento SL, Surita FG, Cecatti JG. Physical exercise during pregnancy: a systematic review. Curr Opin Obstet Gynecol. 2012;24(6):387-394. doi:10.1097/ GCO.0b013e328359f131

(C) 2018 The Author (s); This is an open-access article distributed under the terms of the Creative Commons Attribution License (http://creativecommons.org/licenses/by/4.0), which permits unrestricted use, distribution, and reproduction in any medium, provided the original work is properly cited. 